\title{
Un nuevo enfoque en pos del sueño seguro de los lactantes
} A new approach to safe infant sleep

La muerte inesperada de un lactante durante el sueño continúa desafiando nuestros conocimientos y prácticas. La comunidad científica internacional ha avanzado mucho en la comprensión de sus mecanismos y en el conocimiento de los factores de riesgo que se deben evitar. Pero después del gran descenso en las tasas de mortalidad ocurrido con las primeras campañas en los años 90, desde el año 2000 aproximadamente, no hay mayores cambios. Apenas una leve disminución de los casos con diagnóstico de síndrome de muerte súbita del lactante acompañada también por un leve aumento en los diagnósticos de sofocación o estrangulamiento. ${ }^{1}$

Aunque se puede decir que los registros son deficientes, que los diagnósticos son imprecisos, que no se estudia debidamente el escenario de la muerte y que la autopsia es excepcional, estas falencias han existido siempre, desde hace muchos años y afectan la precisión de las cifras antes y ahora, con distintos matices según los países.

El modelo del triple riesgo, aunque imperfecto como toda idea simplificada, nos permite enfocar en forma esquemática el problema y su situación actual.

\section{¿Qué sabemos hoy?}

1. Que un niño vulnerable, durante el primer año de vida, expuesto a un factor de riesgo ambiental, puede morir durante el sueño por una falla en el mecanismo del microdespertar ante la asfixia.

2. Que un niño vulnerable es el que no tuvo su mejor crecimiento y desarrollo durante la vida intrauterina por lo cual sus centros neurológicos relacionados con la respuesta ante la asfixia son más inmaduros. Es el caso de los niños con hipoxia crónica intrauterina por hipertensión, insuficiencia placentaria, exposición a tabaco, alcohol o drogas ilícitas, estrés, embarazo mal controlado y también causas genéticas que afectan los mecanismos de neurotransmisión.

3. Que el primer año de vida es la etapa de más rápido crecimiento y desarrollo neurológico por lo cual se destaca la importancia de la lactancia materna, el control en salud y la vacunación completa.
4. Que los factores de riesgo son la posición prona, la presencia de objetos sueltos o blandos junto al niño, el sobrecalentamiento y la exposición al tabaco, el alcohol y las drogas.

5. Que algunas medidas recomendadas para la disminución del riesgo, son motivo de controversias: uso del chupete, práctica del colecho, uso de posicionadores o dispositivos para dormir en la cama de los padres. ${ }^{2}$

\section{De la teoría a la práctica}

La primera pregunta es por qué sabiendo todo esto, no conseguimos bajar la mortalidad en los últimos años.

Tendríamos que cuidar más el embarazo para que los niños nazcan con menos vulnerabilidad. Pero la realidad nos muestra un aumento de embarazos en adolescentes, en el consumo de drogas, tabaco y alcohol entre las mujeres jóvenes, un crecimiento del número de personas en condiciones socioeconómicas desfavorables, el progreso de la fertilización asistida en mujeres con dificultades para lograr y mantener una gestación y tantas otras realidades de la vida actual que afectan la etapa del embarazo.

Todos los esfuerzos que se hagan para favorecer el crecimiento y el desarrollo de los niños durante su primer año de vida, no solamente son beneficiosos en el tema que nos ocupa sino que hacen a la mejor oportunidad de lograr una vida saludable: promover $\mathrm{y}$, proteger la lactancia materna, facilitar el acceso de todos los niños a un esquema completo de vacunación, asegurar los controles del niño sano y la atención adecuada en la enfermedad. ${ }^{3}$

Con los factores de riesgo estamos en un problema o más bien, en varios problemas. Uno es la incertidumbre. No siempre podemos identificar al niño vulnerable. Un recién nacido de término, sano, puede ser vulnerable por causa genética, si tiene una deficiencia de la síntesis, liberación o recaptación de sus neurotransmisores. Por eso las recomendaciones sobre sueño seguro son iguales para todos los niños.

Otro aspecto es el desconocimiento por parte del equipo de salud. Si entre los recién nacidos normales en una maternidad, puede haber algún niño vulnerable, en las unidades de cuidados intensivos neonatales, prácticamente todos los son.

Los niños prematuros, de bajo peso, con 
restricción del crecimiento intrauterino, con síndromes genéticos, con patología grave neonatal, son claramente vulnerables. Las familias de estos niños deberían ser capacitadas, entre otros temas, para brindarles un sueño seguro. Este rol corresponde al equipo de salud. El personal de enfermería comparte mucho tiempo con las familias durante la internación; son los cuidadores directos para los recién nacidos y los modelos indiscutidos para los padres. Sin embargo, algunos profesionales no conocen las recomendaciones para el sueño seguro, otros las conocen pero no las ponen en práctica. ${ }^{4}$ Lo mismo se puede decir de las familias. ${ }^{5}$

Acá es donde se plantea la necesidad de cuestionarse qué podemos hacer para dar alguna respuesta a estas situaciones.

El proyecto GAPS (Global Action and Prioritization of Sudden Infant Death Project), fue un proceso de consenso internacional que comenzó en 2015 con el objetivo de definir prioridades en el desarrollo de investigaciones para disminuir la tasa de muertes inesperadas de los lactantes. ${ }^{6}$

Luego de tres rondas de discusión se llegó a la conclusión que necesitamos:

1. Comprender mejor los mecanismos biológicos involucrados en la muerte inesperada y su interacción con los factores ambientales, en diferentes edades y etapas de la maduración.

2. Desarrollar buenas prácticas en la recolección, manejo y difusión de datos.

3. Identificar mejor a las poblaciones más vulnerables y tener una comunicación más efectiva de los riesgos en la medida que factores sociales y culturales determinen la elección familiar de prácticas de sueño compartido.

La actualización 2016 de las recomendaciones para un medio ambiente de sueño seguro para los lactantes de la Academia Americana de Pediatría, incorporó nuevas evidencias sobre el contacto piel a piel en el recién nacido, el uso de cunas de colecho y dispositivos para el sueño en la cama de los padres, el sueño en sillones o sofás y en asientos, y el uso de ropa de cama suelta después de los 4 meses.

En consonancia con las nuevas tendencias sobre la forma de comunicar estas recomendaciones, se pone énfasis en tener un diálogo abierto, sin juzgamientos sobre las prácticas de sueño de las familias. Las situaciones particulares de cada una de ellas requieren un mensaje personalizado que tenga en cuenta los riesgos y beneficios relativos. ${ }^{7}$

Estamos dejando atrás el mensaje terminante para ingresar en el proceso de decisiones compartidas, donde se toman en cuenta las evidencias científicas, dentro de un contexto personal y familiar que incluye lo social, lo económico y lo cultural. ${ }^{8}$

Todos estos aspectos influyen en la forma en que cada familia cuida a sus hijos. Ser inflexible en los mandatos genera alejamiento. Un diálogo abierto, que tenga en cuenta las creencias y situación de cada familia, permitirá sugerir la mejor forma de disminuir el riesgo dentro de sus posibilidades.

Naturalmente esto lleva más tiempo pero parece el mejor camino a seguir.

Dra. Norma Elena Rossato Grupo de Trabajo "Trastornos del sueño y muerte súbita e inesperada del lactante"

http: / / dx.doi.org/10.5546/ aap.2019.354

Texto completo en inglés:

http:/ / dx.doi.org/10.5546/aap.2019.eng.354

Cómo citar: Rossato NE.Un nuevo enfoque en pos del sueño seguro de los lactantes. Arch Argent Pediatr 2019;117(6):354-355.

\section{REFERENCIAS}

1. Moon RY; Task Force On Sudden Infant Death Syndrome. SIDS and Other Sleep-Related Infant Deaths: Evidence Base for 2016 Updated Recommendations for a Safe Infant Sleeping Environment. Pediatrics. 2016; 138(5):e20162940.

2. Task Force On Sudden Infant Death Syndrome. SIDS and Other Sleep-Related Infant Deaths: Updated 2016 Recommendations for a Safe Infant Sleeping Environment. Pediatrics. 2016; 138(5):e20162938.

3. Hauck FR, Tanabe KO. Beyond "Back to Sleep": Ways to Further Reduce the Risk of Sudden Infant Death Syndrome. Pediatr Ann. 2017; 46(8):e284-90.

4. Newberry JA. Creating a Safe Sleep Environment for the Infant: What the Pediatric Nurse Needs to Know. J Pediatr Nurs. 2019; 44:119-22.

5. Hwang SS, Corwin MJ.Safe Infant Sleep Practices: Parental Engagement, Education, and Behavior Change. Pediatr Ann. 2017; 46(8):e291-6.

6. Hauck FR, McEntire BL, Raven LK, Bates FL, et al. Research Priorities in Sudden Unexpected Infant Death: An International Consensus. Pediatrics. 2017;140(2):e20163514.

7. Altfeld S, Peacock N, Rowe HL, Massino J, et al. Moving Beyond "Abstinence-Only" Messaging to Reduce SleepRelated Infant Deaths. J Pediatr. 2017; 189:207-12.

8. Gray B, Coker TR. When shared decision-making and evidence based practice clash: Infant sleep practices. J Paediatr Child Health. 2019 Jul 29. doi: 10.1111/jpc.14577. [Epub ahead of print] 